\title{
Evaluation of the prognostic and physiological functions of death associated protein kinase 1 in breast cancer
}

\author{
YIDE HUANG ${ }^{1}$, MEIZHEN LIN $^{1},{\text { XIANGJIN } \mathrm{CHEN}^{2}, \text { CHAOQUN HUANG }^{1}, \text { XIULI ZHANG }}^{1}$, \\ LING CHEN $^{2}$, KUNLIN WU ${ }^{2}$, YUPENG CHEN ${ }^{3}$, YOUZHI ZHU ${ }^{2}$ and YAO LIN ${ }^{1}$ \\ ${ }^{1}$ Provincial University Key Laboratory of Cellular Stress Response and Metabolic Regulation, College of Life Sciences, \\ Fujian Normal University, Fuzhou, Fujian 350117; Departments of ${ }^{2}$ Thyroid and Breast Surgery and ${ }^{3}$ Pathology, \\ The First Affiliated Hospital of Fujian Medical University, Fuzhou, Fujian 350000, P.R. China
}

Received July 28, 2016; Accepted December 5, 2017

DOI: $10.3892 / 01.2018 .8439$

\begin{abstract}
Death associated protein kinase 1 (DAPK1) is a notable serine/threonine kinase involved in the regulation of multiple cellular pathways, including apoptosis and autophagy. Although DAPK1 is usually considered to be a tumor suppressor, it was previously reported to promote the viability of p53 mutant cancer cell lines and possess physiological oncogenic functions in breast cancer. However, the ability of endogenous DAPK1 to suppress breast cancer cell mobility has not been assessed. In the present study, the prognostic function of DAPK1 in a Chinese patient cohort was evaluated, and no significant association was observed between DAPK1 expression and patient survival or lymph node metastasis. In order to investigate the physiological function of endogenous DAPK1, stable inducible DAPK1 knockdown MCF7 and MDA-MB-231 cell lines were established. Consistent with previous studies, endogenous DAPK1 only regulated cell viability in p53 mutant MDA-MB-231 cells. However, knockdown of DAPK1 did not significantly affect cell motility of either MCF7 or MDA-MB-231 cells. Altogether, these results further explored the function of endogenous DAPK1 in breast cancer and may shed light in understanding the molecular signaling pathways regulating the physiological function of DAPK1.
\end{abstract}

Correspondence to: Dr Yao Lin, Provincial University Key Laboratory of Cellular Stress Response and Metabolic Regulation, College of Life Sciences, Fujian Normal University, Qishan Campus, 1 Keji Road, Shangjie, Minhou, Fuzhou, Fujian 350117, P.R. China E-mail: yaolin@fjnu.edu.cn

Dr Youzhi Zhu, Department of Thyroid and Breast Surgery, The First Affiliated Hospital of Fujian Medical University, 20 Chazhong Road, Fuzhou, Fujian 350000, P.R. China

E-mail: zhu1755@yeah.net

Key words: death associated protein kinase 1, breast cancer, migration, proliferation, invasion

\section{Introduction}

Death associated protein kinase 1 (DAPK1) is a notable multifunctional protein kinase involved in the regulation of apoptosis, autophagy, membrane blebbing and inflammation (1-4). Conventionally, DAPK1 is considered to be a tumor suppressor, and its expression is often downregulated in various types of cancer, including leukemia, lung cancer and ovarian cancer due to DNA methylation (5). In 2015, Zhao et al (6) reported that endogenous DAPK1 functioned as a survival factor in p53-mutant cancer types, particularly triple negative breast cancers (TNBC). This oncogenic function of DAPK1 was mediated by its stimulatory effect towards mammalian target of rapamycin complex 1 (mTORC1) and subsequent proliferation induction (6), which was consistent with a previous study demonstrating that DAPK1 may activate mTORC1 via tuberous sclerosis 2 (TSC2) phosphorylation (7). However, the effect of DAPK1 on colony formation, migration or invasion of breast cancer cells were not evaluated (6). DAPK1 has been demonstrated to inhibit cell mobility by inhibiting the integrin-mediated polarity pathway (8). Therefore, it is important to examine the functions of DAPK1 aside from proliferation induction in p53 wild-type and mutant breast cancer cells.

In the present study, the prognostic value of DAPK1 was evaluated using a Chinese breast cancer patient cohort. The regulatory function of endogenous DAPK1 for breast cancer cell viability, colony formation and migration was assessed using a stable inducible knockdown system.

\section{Materials and methods}

Cell culture. Human breast cancer epithelial cell lines MCF7 [estrogen receptor (ER)-positive] and MDA-MB-231 derived from a metastatic site pleural effusion were purchased from the Institute of Biochemistry and Cell Biology at the Chinese Academy of Sciences (Shanghai, China). Both of MCF7 and MDA-MB-231 cell lines were verified using short tandem repeat genotyping and tested negative for mycoplasma. The MCF7 cells were maintained in RPMI-1640, whereas MDA-MB-231 cells were maintained in Dulbecco's modified Eagle's medium (DMEM; both from Invitrogen; Thermo Fisher Scientific, Inc., Waltham, MA, USA), containing 
$10 \%$ fetal bovine serum (FBS; Equitech-Bio, Inc., Kerrville, TX, USA), $100 \mathrm{U} / \mathrm{ml}$ penicillin $\mathrm{G}$, and $100 \mu \mathrm{g} / \mathrm{ml}$ streptomycin (Invitrogen; Thermo Fisher Scientific, Inc.). All cell lines were incubated at $37^{\circ} \mathrm{C}$ in a humidified incubator with $5 \% \mathrm{CO}_{2}$.

Clinical samples. A total of 66 tissue samples (with a median age of 49 and an age range of 26 to 77 years old) from patients with breast cancer were obtained from patients with invasive ductal carcinoma at The First Affiliated Hospital of Fujian Medical University (Fuzhou, China). All patients were admitted between January 2010 and December 2010. The pathological and clinical data including histological grade, invasion, and metastasis were collected for all samples. Evaluation of the statuses of ER, progesterone receptor and human epidermal growth factor receptor- 2 was performed during patient recruitment by the Pathology Department of The First Affiliated Hospital of Fujian Medical University. The present study was approved by the Ethics Committee of the First Affiliated Hospital of Fujian Medical University and written informed consent was obtained from all patients involved.

Immunohistochemistry (IHC) analysis. Sample preparation and IHC staining was performed as previously described (9). Briefly, IHC staining for death-associated protein kinase (DAPK) was performed on formalin-fixed, paraffin-embedded in tissue sections (4- $\mu$ m-thick) The sections were deparaffinized with $100 \%$ dimethylbenzene and rehydrated through $100,100,95,85$, and $75 \%$ ethanol series. Endogenous peroxidase was blocked by incubation in $3 \% \mathrm{H}_{2} \mathrm{O}_{2}$ for 10 mins at room temperature. The sections were then washed in PBS and blocked with $10 \%$ goat serum (Beijing Zhongshan Jinqiao Biotechnology Co., Ltd., Beijing, China) for 30 mins and incubated with anti-DAPK primary antibody (cat. no. 3008; 1:150 dilution; Cell Signaling Technology, Inc., Danvers, MA, USA) in a humidified chamber at $4^{\circ} \mathrm{C}$ overnight. Following 3 additional washes in PBS, the sections were incubated with HRP-conjugated secondary antibody (cat. no. sc-2031; 1:2,000 dilution; Santa Cruz Biotechnology, Inc. TX, USA) for $30 \mathrm{mins}$ at room temperature. The visualization signal was developed with diaminobenzidine (DAB) solution and all slides were counterstained with $20 \%$ hematoxylin for 30 s at room temperature. Finally, all slides were dehydrated using a series of ethanol (85\% ethanol for $5 \mathrm{~min}, 95 \%$ ethanol for $5 \mathrm{~min}$ and $100 \%$ ethanol for $5 \mathrm{mins}$ ) and mounted on cover slips. The IHC-stained tissue sections were reviewed with a light microscope (BX51; Olympus Corporation, Tokyo, Japan) at magnifications, x200 and x400. The staining intensity was classified as negative, and positive. Briefly, the protein expression was scored independently according to the intensity of cellular staining and the proportion of stained tumor cells. The staining intensity was scored as 0 , no staining; 1 , weak staining, light yellow; 2 , moderate staining, yellow brown; and 3 , strong staining, brown. The proportions of stained tumor cells were classified as $0, \leq 5 \%$ positive cells; $1,6-25 \%$ positive cells; $2,26-50 \%$ positive cells; and $3, \geq 51 \%$ positive cells. The total scores for intensity and proportion were used to signify the level of protein expression. A score of 3 or less was considered negative DAPK expression, and a score of 4 or more was considered positive DAPK expression.
Construction of stable inducible cell lines. Target cells (MCF7 and MDA-MB-231) were seeded at a density of $1 \times 10^{5}$ cells/ml into $24-w e l l$ plates. Subsequent to growing to $50-70 \%$ confluence, cells were infected with $250 \mu \mathrm{l}$ short hairpin RNA (shRNA) viruses mixed with serum-free culture medium (RPMI-1640 for MCF 7, DMEM for MDA-MB-231). A total of $2 \mathrm{~h}$ later, $250 \mu 1$ 10\% FBS normal culture media was added. After $48 \mathrm{~h}$, cells were cultured for 2 days with screening culture medium containing $0.4 \mu \mathrm{g} / \mathrm{ml}$ puromycin, which was used to select for infected cells to further culture. The stable inducible cell lines were successfully established.

Western blot analysis. Cells were homogenized in 100-200 $\mu \mathrm{l}$ radioimmunoprecipitation assay lysis buffer (Beyotime Institute of Biotechnology, Shanghai, China) containing protease inhibitors (Roche Diagnostics, Basel, Switzerland) and incubated at $4^{\circ} \mathrm{C}$ for $30 \mathrm{~min}$. Protein concentrations were measured using the Micro BCA protein assay kit (Pierce; Thermo Fisher Scientific, Inc.). Cell lysate (60 $\mu \mathrm{g}$ per lane) was separated on a $10 \%$ SDS-PAGE gel and subsequently transferred to a Hybond $\mathrm{C}$ nitrocellulose membrane (Thermo Fisher Scientific, Inc.). The nitrocellulose membrane was blocked with $5 \%$ fat-free milk in TBS-T buffer (20 mM Tris- $\mathrm{HCl} \mathrm{pH} 8.0$, $150 \mathrm{mM} \mathrm{NaCl}, 0.1 \%$ Tween-20) for $1 \mathrm{~h}$ at room temperature and subsequently incubated overnight at $4^{\circ} \mathrm{C}$ with the primary antibodies anti-human DAPK (1:1,000 dilution; cat. no. 3008; Cell Signaling Technology, Inc.) and anti-human GAPDH (1:5,000 dilution; cat. no. 2118; Cell Signaling Technology, Inc.), then washing (3x $10 \mathrm{~min}$ ) in TBS-T buffer, followed by incubating for $1 \mathrm{~h}$ at room temperature with IRDye $800 \mathrm{CW}$-conjugated goat-anti-Rabbit secondary antibodies (cat. no. C60607-15; LI-COR Biosciences, Lincoln, NE, USA). The membrane was finally washed (3x 10 min) in TBS-T buffer again. The signals were detected and measured using LICOR Odyssey system (LI-COR, Nebraska, USA).

Sulforhodamine $B(S R B)$ assay. Cells were first seeded in 6-cm dishes with or without $400 \mathrm{ng} / \mathrm{ml}$ doxycycline (Dox) (cat. no. 324385; EMB Millipore, Billerica, MA, USA) and cultured for 5 days. Then, for the SRB assay, MCF7 and MDA-MB-231 cells were seeded at a density of $7 \times 10^{3}$ and $1 \times 10^{4}$ cells/well into 96-well plates, respectively. Next, cells continued to grow with or without Dox for another 96 h. Subsequent to culturing, total cell amounts were detected using an Sulforhodamine B (cat. no. S1402; Sigma-Aldrich; Merck KGaA, Darmstadt, Germany) assay as previously described (10).

Colony formation. Cells were first seeded in $6 \mathrm{~cm}$ dishes with or without Dox and allowed to grow for 5 days. Then for the colony formation assay, MCF7 and MDA-MB-231 cells were seeded at a density of 500 cells/well into six-well plates. Next, cells continued to grow with or without Dox for 7 days. Following culturing, the number of clones formed was detected using a colony formation assay as previously described (10).

Cell migration assays. Cells were first seeded in $6 \mathrm{~cm}$ dishes with or without Dox for 5 days. Cells were then seeded in six-well plates with or without Dox and allowed to grow to confluence. The cell migration was then measured using a 
Table I. General profile of 66 patients with invasive breast ductal carcinoma.

\begin{tabular}{|c|c|}
\hline Characteristics & Number of patients/total (\%) \\
\hline \multicolumn{2}{|l|}{ Age (years) } \\
\hline$<55$ & $41 / 66(62.1)$ \\
\hline$\geq 55$ & 25/66 (37.9) \\
\hline \multicolumn{2}{|c|}{ Histological grade } \\
\hline I & $3 / 66(4.5)$ \\
\hline II & $53 / 66(80.3)$ \\
\hline III & $10 / 66(15.2)$ \\
\hline \multicolumn{2}{|c|}{ Axillary nodal status } \\
\hline Negative & 25/66 (37.9) \\
\hline Positive & $33 / 66(50.0)$ \\
\hline NA & $8 / 66(12.1)$ \\
\hline \multicolumn{2}{|l|}{ ER } \\
\hline Negative & 29/66 (43.9) \\
\hline Positive & $31 / 66(46.9)$ \\
\hline NA & $6 / 66(9.2)$ \\
\hline \multicolumn{2}{|l|}{ PR } \\
\hline Negative & $38 / 66(57.5)$ \\
\hline Positive & 22/66 (33.3) \\
\hline NA & 6/66 (9.2) \\
\hline \multicolumn{2}{|l|}{ Her2 } \\
\hline Negative & $30 / 66(45.4)$ \\
\hline Positive & $30 / 66(45.4)$ \\
\hline NA & $6 / 66(9.2)$ \\
\hline \multicolumn{2}{|l|}{ DAPK } \\
\hline Positive & $53 / 66(80.3)$ \\
\hline Negative & $13 / 66(19.7)$ \\
\hline
\end{tabular}

NA, not available; ER, estrogen receptor; PR, progesterone receptor; Her2, human epidermal growth factor receptor-2; DAPK, death associated protein kinase.

scratch wound healing assay as previously described (9). Briefly, the monolayer cells were scratched with a $10 \mu \mathrm{l}$ sterile pipette tip to create a $\sim 10 \mu \mathrm{m}$ wound, washed twice with serum-free media to remove floating cells, and left to grow in serum-free media. The cells migrating from the leading edge were photographed at 0 and $24 \mathrm{~h}$. Multiple views of each well were documented with an inverted light microscope (CKX-41; Olympus Corporation, Tokyo, Japan) at magnification, x200 and three independent experiments were performed. The percentage of reduced cover area was calculated as $(\mathrm{g} 0-\mathrm{gt}) / \mathrm{g} 0$ $\mathrm{x} 100 \%$. The g0 and gt were the wound width at 0 and $24 \mathrm{~h}$, respectively. For the cell migration assay, Transwell chambers (polycarbonate filters of $8 \mu \mathrm{m}$ porosity; BD Biosciences, Franklin Lakes, NJ, USA) were used. The bottom chamber was filled with a culture medium (RPMI-1640 for MCF 7 cells and DMEM for MDA-MB-231 cells) containing 10\% FBS and the upper chamber was filled with serum-free medium. A total of $2 \times 10^{5}$ stable cells were suspended in serum-free medium and plated in the upper chamber. Following incubation for $24 \mathrm{~h}$,
Table II. Association between DAPK and other clinicopathological characteristics.

\begin{tabular}{|c|c|c|c|}
\hline \multirow[b]{2}{*}{ Characteristics } & \multicolumn{2}{|c|}{ DAPK } & \multirow[b]{2}{*}{ P-value } \\
\hline & Negative & Positive & \\
\hline Age (years) & & & 0.185 \\
\hline$<55$ & 6 & 35 & \\
\hline$\geq 55$ & 7 & 18 & \\
\hline Histological grade & & & $0.036^{\mathrm{a}}$ \\
\hline I & 2 & 1 & \\
\hline II & 11 & 42 & \\
\hline III & 0 & 10 & \\
\hline Axillary nodal status & & & 0.910 \\
\hline Negative & 5 & 20 & \\
\hline Positive & 7 & 26 & \\
\hline ER & & & 0.438 \\
\hline Negative & 7 & 22 & \\
\hline Positive & 5 & 26 & \\
\hline PR & & & 0.688 \\
\hline Negative & 7 & 31 & \\
\hline Positive & 5 & 17 & \\
\hline Her2 & & & 0.519 \\
\hline Negative & 5 & 25 & \\
\hline Positive & 7 & 23 & \\
\hline
\end{tabular}

${ }^{a} \mathrm{P}<0.05$. ER, estrogen receptor; $\mathrm{PR}$, progesterone receptor; Her2, human epidermal growth factor receptor-2; DAPK, death associated protein kinase.

the cells were removed from the upper chamber using a cotton swab. Cells that had penetrated and attached to the bottom of the filter were fixed for $10 \mathrm{~min}$ at room temperature with $4 \%$ formaldehyde in PBS, followed by 20 min staining with $0.5 \%$ crystal violet at room temperature and then subjected to imaging under a x20 objective lens and photographed. Statistical results of cell numbers per each image field were obtained from three independent experiments averaging from five image fields.

Statistical analysis. Analyses of the association between the protein expression of DAPK1 and clinicopathological variables were performed using Fisher's exact test. Progression-free survival was defined as the interval from the first day of surgery until tumor progression, mortality or the end of follow-up. Time-to-progress comparisons were performed using a Log-rank test. Univariate Cox-regression and multivariate analysis were additionally performed for available clinicopathological parameters. Physiological functional data of DAPK1 in breast cancer were analyzed using GraphPad Prism version 7.0 software (Graphpad Software, Inc., La Jolla, CA, USA). All data are presented as the mean \pm standard deviation of three independent experiments. The significance of difference in colony formation and cell migration of MCF7 and MDA-MB-231 cells with or without 
A

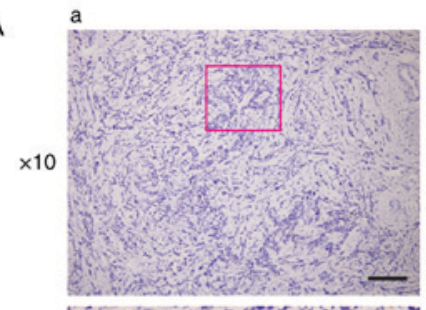

b
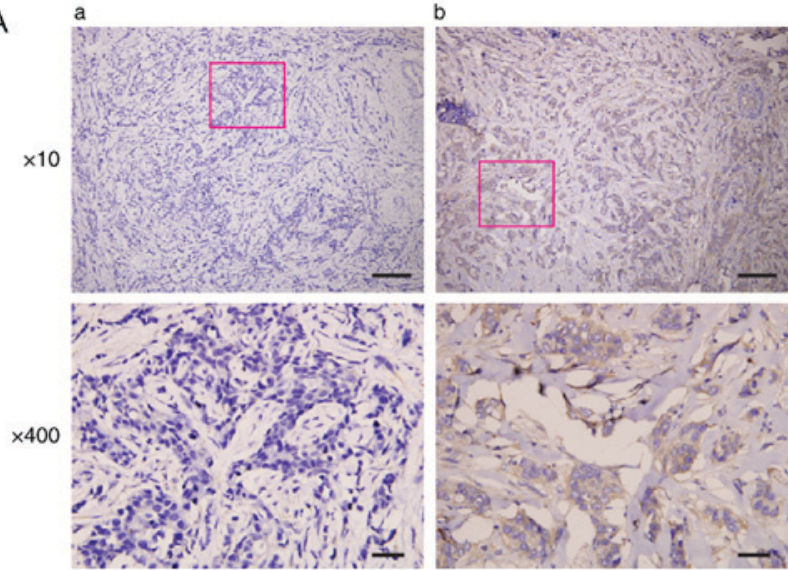
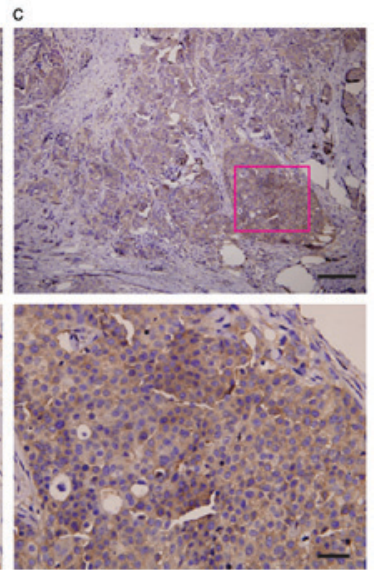

B

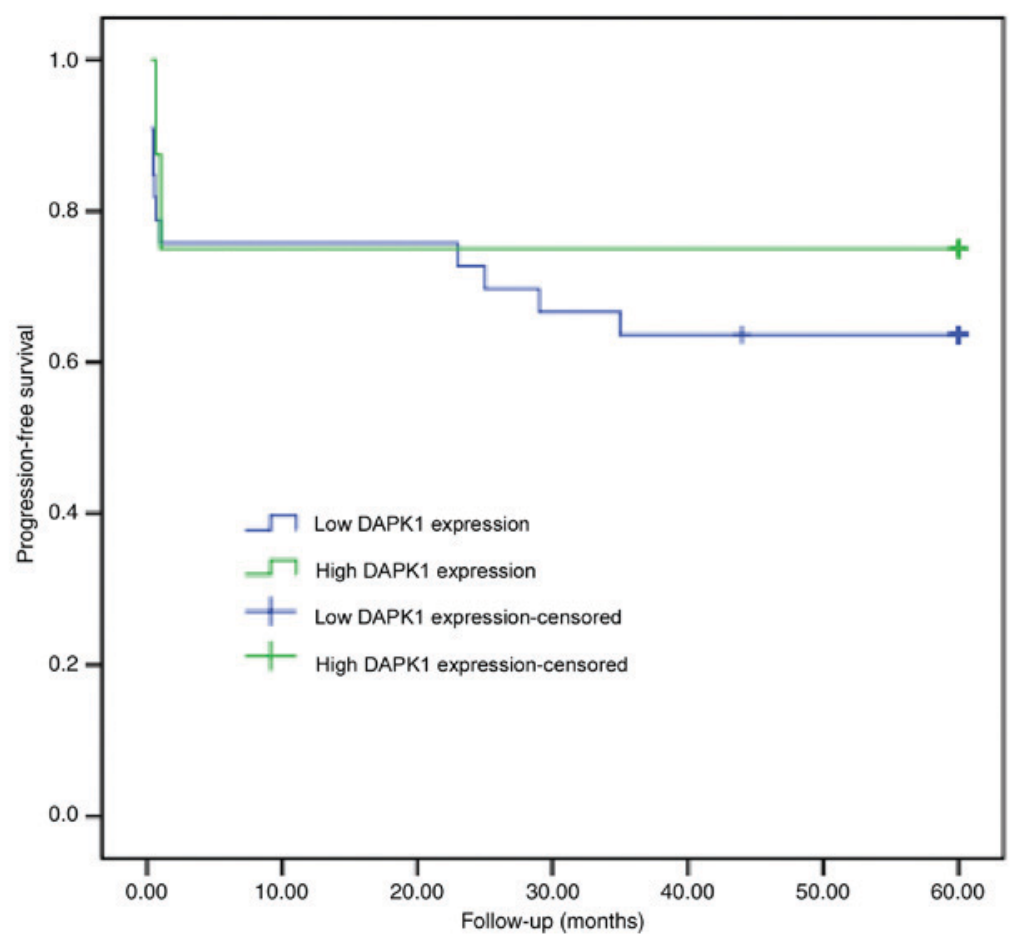

Figure 1. DAPK protein expression detected using IHC staining did not correlate with patient survival. (A) Representative IHC staining of DAPK protein in breast cancer patient samples. The DAPK protein staining was categorized as (a) negative, (b) weakly positive and (c) strongly positive. All representative images were taken using a light microscope at magnification, $\mathrm{x} 10$ (bars=100 $\mu \mathrm{m}$ ) and x40 (bars=20 $\mu \mathrm{m}$ ). (B) Association between DAPK and the 5-year progression free survival of breast cancer patients. The association between DAPK expression and the 5-year progression free survival times was analyzed using Kaplan-Meier survival analysis $(\mathrm{P}=0.6)$. The Kaplan Meier curve differences were compared by Log-Rank test. DAPK, death associated protein kinase; IHC, immunohistochemistry.

Dox induction were examined using a two-tailed Student's t-test. Multiple comparisons were performed using one-way analysis of variance followed by Dunnett's post-hoc test. For all analyses, $\mathrm{P}<0.05$ was considered to indicate a statistically significant difference.

\section{Results}

Association between DAPKI expression and clinicopathological characteristics of patients with breast cancer. First, the prognostic function of DAPK1 in breast cancer was evaluated using the IHC staining of 66 samples from patients with breast cancer (Fig. 1A). The general profile of this patient cohort was listed on Table I. Of all the clinicopathological parameters available, DAPK1 expression was only significantly associated with histological grade $(\mathrm{P}=0.036$; Table II). Additionally, when patients were grouped as ER-positive and ER-negative, DAPK1 expression was only significantly associated with histological grade in ER-negative patients ( $\mathrm{P}=0.042$; Table III). Furthermore, DAPK1 expression was not significantly associated with the 5-year progression-free survival of patients with breast cancer (Fig. 1B). These data demonstrated that despite the previous study that observed that DAPK1 may function as a survival factor in breast cancer to promote cell growth (6), DAPK1 did not significantly affect the overall survival rate in the present cohort. Unfortunately, the p53 mutation was not routinely examined at Fujian Medical Hospital. Therefore, it was difficult to distinguish if p53 status affected the prognostic value of DAPK1 in the present cohort. 
Table III. Association between DAPK and other clinicopathological characteristics in ER positive and negative patient samples.

\begin{tabular}{|c|c|c|c|c|c|c|}
\hline \multirow[b]{2}{*}{ Characteristics } & \multicolumn{2}{|c|}{ ER-negative } & \multirow[b]{2}{*}{ P-value } & \multicolumn{2}{|c|}{ ER-positive } & \multirow[b]{2}{*}{ P-value } \\
\hline & DAPK-negative & DAPK-positive & & DAPK-negative & DAPK-positive & \\
\hline Age (years) & & & 0.403 & & & 0.998 \\
\hline$<55$ & 3 & 14 & & 3 & 17 & \\
\hline$\geq 55$ & 4 & 8 & & 2 & 9 & \\
\hline Histological grade & & & 0.066 & & & $0.042^{\mathrm{a}}$ \\
\hline I & 0 & 0 & & 2 & 1 & \\
\hline II & 7 & 13 & & 3 & 24 & \\
\hline III & 0 & 9 & & 0 & 1 & \\
\hline Axillary nodal status & & & 0.997 & & & 0.998 \\
\hline Negative & 2 & 7 & & 2 & 12 & \\
\hline Positive & 4 & 11 & & 3 & 11 & \\
\hline PR & & & 0.998 & & & 0.368 \\
\hline Negative & 6 & 19 & & 1 & 12 & \\
\hline Positive & 1 & 3 & & 4 & 14 & \\
\hline Her2 & & & 0.382 & & & 0.997 \\
\hline Negative & 1 & 8 & & 4 & 17 & \\
\hline Positive & 6 & 14 & & 1 & 9 & \\
\hline
\end{tabular}

${ }^{a} \mathrm{P}<0.05$. ER, estrogen receptor; PR, progesterone receptor; Her2, human epidermal growth factor receptor-2; DAPK, death associated protein kinase.

A
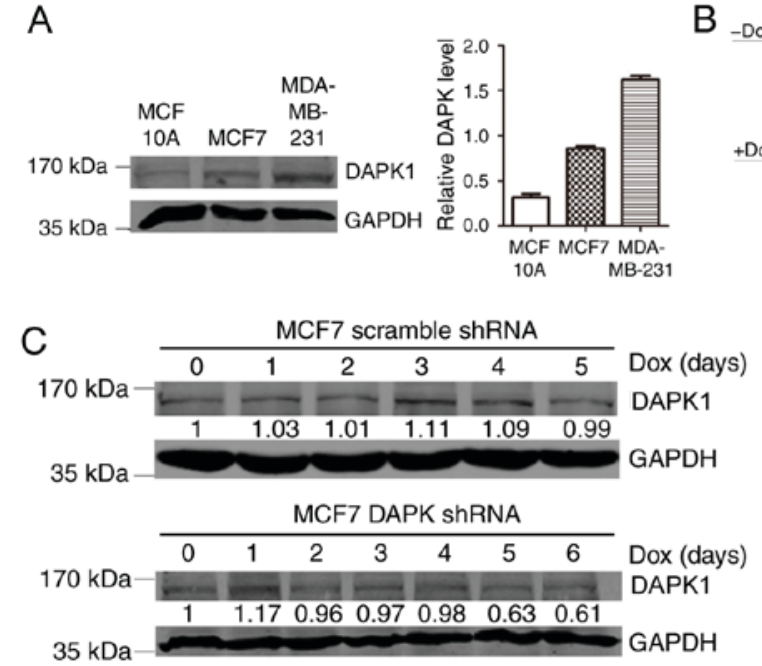

$\mathrm{B}_{-\mathrm{Dox}}$

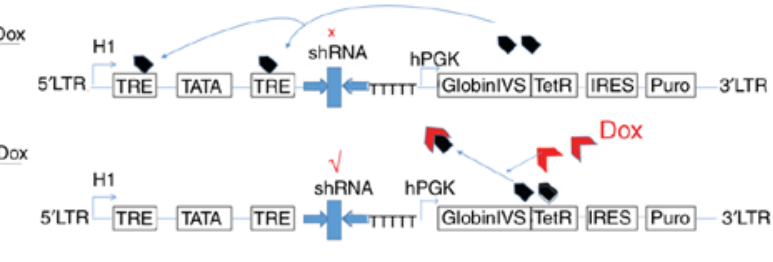

D

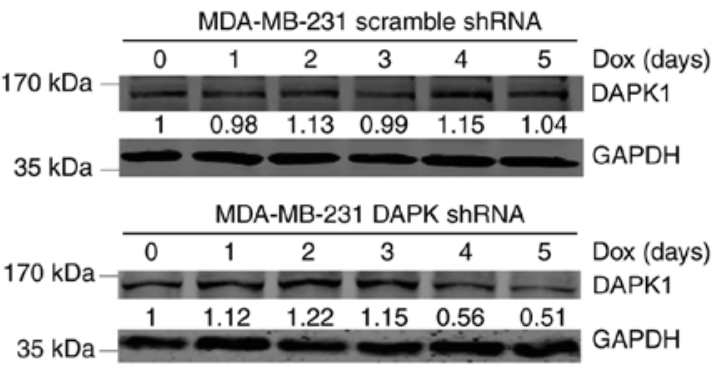

Figure 2. Construction of stable inducible DAPK1-knockdown breast cancer cell lines. (A) Protein expression of DAPK in MCF10A, MCF7 and MDA-MB-231 cells were detected using western blotting (left panel). This experiment was repeated three times and the results were quantified (right panel). (B) Schematic of Dox inducible mechanism. Subsequent to virus infection and puromycin selection, the inducible knockdown effect of the stable (C) MCF and (D) MDA-MB-231 cells was examined using Dox treatment over time. The ratio of DAPK to GAPDH at day 0 was considered to be 1 . The proportions of the DAPK/GAPDH ratios of subsequent days were examined and presented. These experiments were repeated three times. DAPK, death associated protein kinase; Dox, doxycycline.

Expression of DAPK1 in breast cancer cell lines. Next, the functions of DAPK1 in breast cancer cell lines were investigated. The p53 wild-type MCF7 and p53 mutant TNBC MDA-MB-231 cell lines were employed. Compared with the normal breast epithelial cell line MCF10A, MCF7 and MDA-MB-231 cell lines exhibited enhanced DAPK1 expression, suggesting that DAPK1 may be overexpressed in breast cancer cell lines (Fig. 2A).
Effect of DAPK1 knockdown in MCF7 and MDA-MB-231 cells. In order to evaluate the physiological function of DAPK1 in these breast cancer cell lines, a Dox inducible shRNA plasmid was used to create stable inducible DAPK1 knockdown cell lines (Fig. 2B). When exposed to Dox, stable MCF7 and MDA-MB-231 cells revealed substantial DAPK1 downregulation, indicating successful establishment of the 
A

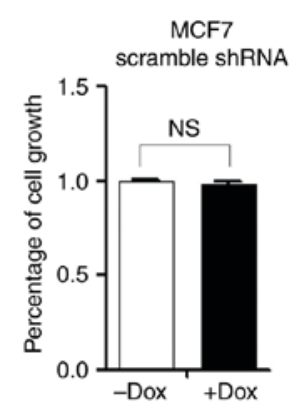

B

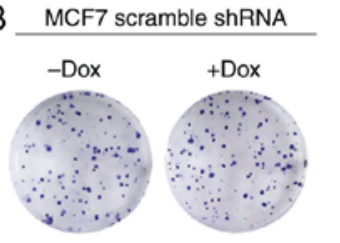

MDA-MB-231 scramble shRNA

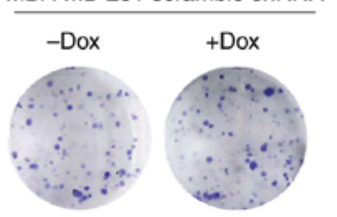

MCF7
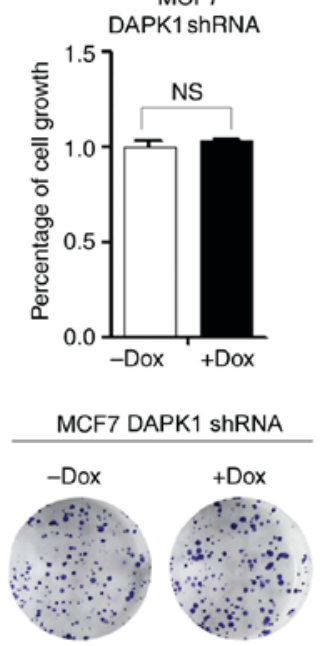

MDA-MB-231 DAPK1 shRNA

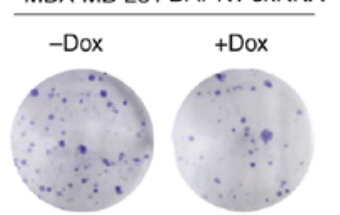

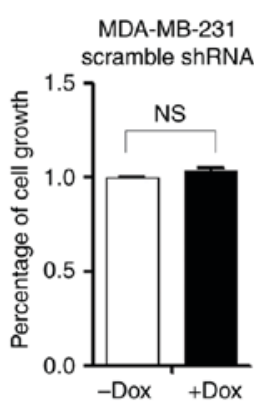
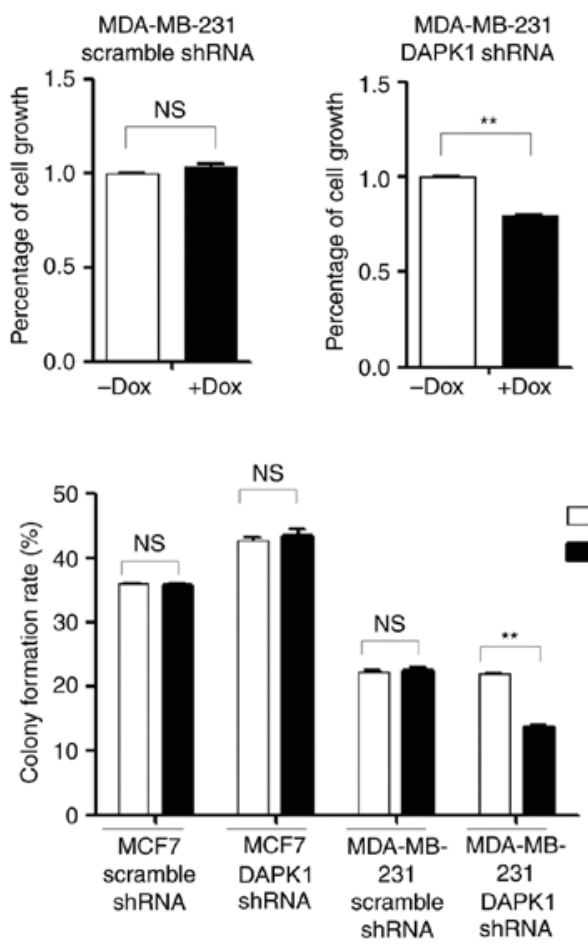

Figure 3. Inducible knockdown of DAPK decreased the viability and colony formation of MDA-MB-231 cells. (A) The stable inducible cells treated with or without Dox were subject to a sulforhodamine B assay. The cells without Dox treatment in each group were considered as 1.0. (B) The stable inducible cells treated with or without Dox were subject to a colony formation assay. The representative images were presented on the left and the quantification on the right panel. All experiments were performed in triplicate. ${ }^{* *} \mathrm{P}<0.01$ with comparisons shown by lines, as determined using a two-tailed Student's t-test. NS, not significant; DAPK, death associated protein kinase; Dox, doxycycline; shRNA, short hairpin RNA.
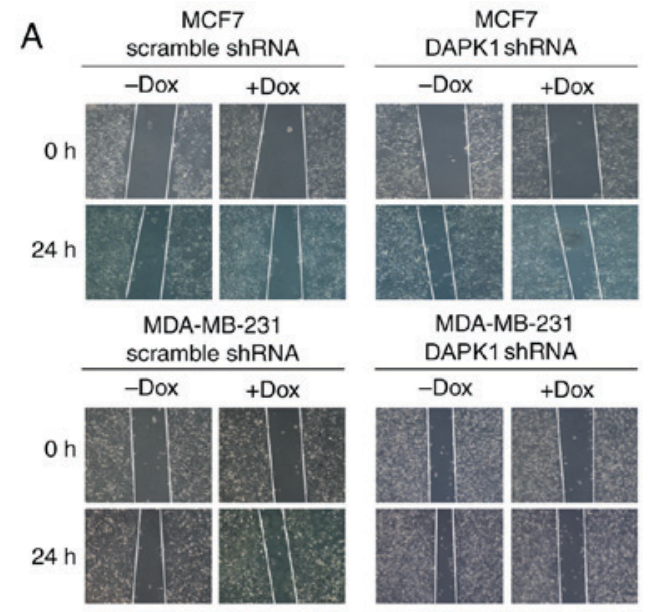

C
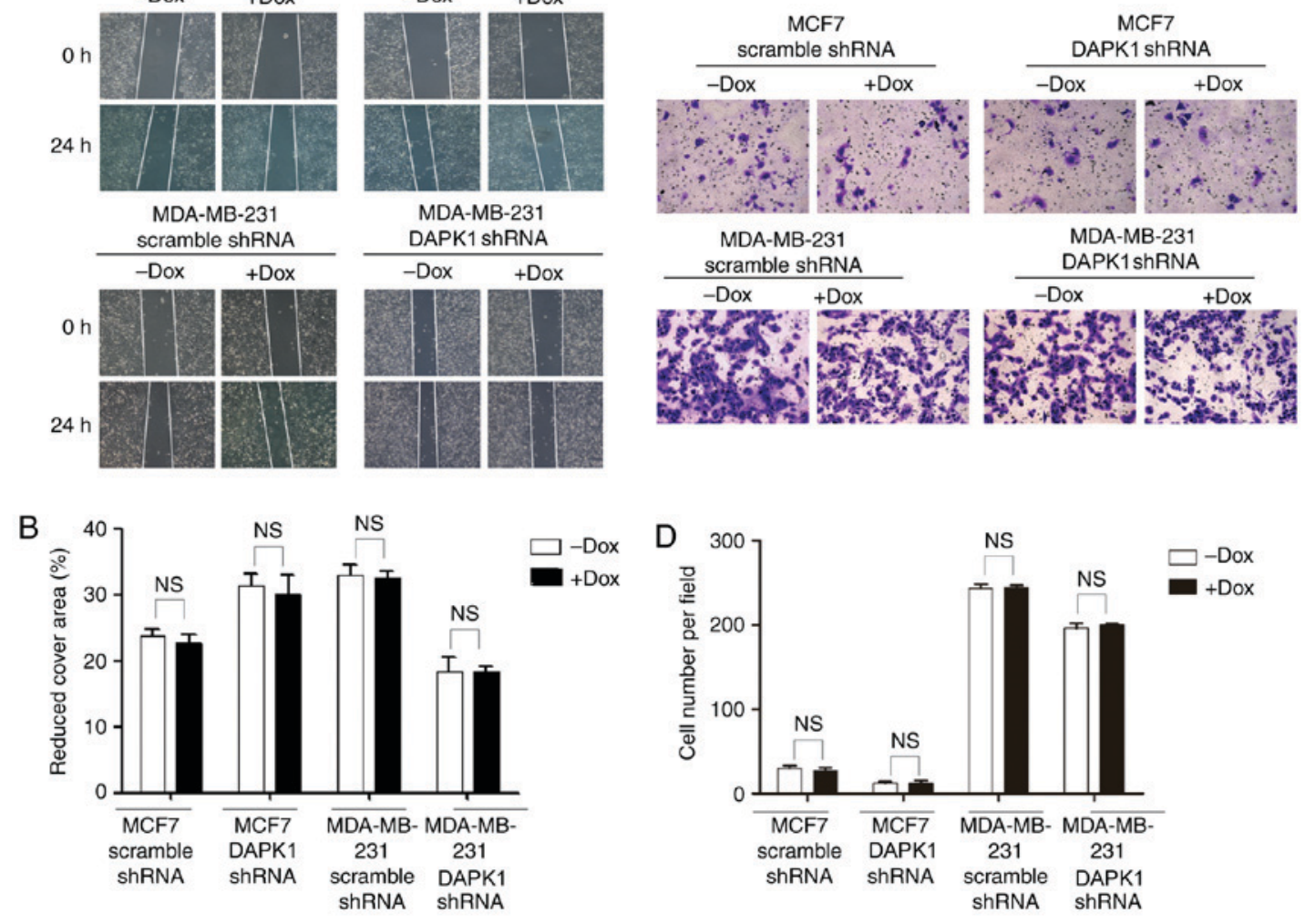

Figure 4. Inducible knockdown of DAPK did not affect cell migration. The stable inducible cells treated with or without Dox were subject to scratch wound healing and Transwell assay experiments. (A) Representative images of scratch wound healing assay were (B) quantified. The percentage of reduced cover area was calculated as (g0-gt)/g0 x100\%. The g0 and gt were the wound width at 0 and $24 \mathrm{~h}$ respectively. (C) Representative images of Transwell experiments were (D) quantified. All experiments were performed in triplicate. NS, not significant; DAPK, death associated protein kinase; Dox, doxycycline; shRNA, short hairpin RNA. 
stable cells (Fig. 2C and D). Next, SRB and colony formation assays were performed to investigate the function of endogenous DAPK1 on cell viability, and colonization ability. The knockdown of DAPK1 only significantly reduced the viability and colony forming ability of MDA-MB-231 cells treated with Dox $(\mathrm{P}<0.01)$, but not MCF7 cells (Fig. 3), consistent with the previous report that DAPK1 only affects breast cancer viability in p53 mutant cells (6). Then, the effect of DAPK1 on cell migration was investigated. Following the knockdown of DAPK1, no significant difference was observed in MCF7 or MDA-MB-231 cells (Fig. 4), suggesting endogenous DAPK1 does not regulate cell motility in these cells.

\section{Discussion}

In the present study, expression of DAPK1 protein was identified in 53/66 samples from patients with breast cancer. The observation that the two breast cancer cell lines exhibited enhanced DAPK1 protein expression in comparison with the normal breast epithelial cell line MCF10A, as demonstrated by western blotting, was concordant with the IHC results. DAPK1 DNA methylation and the loss of its mRNA expression had been previously reported in breast cancer (11). The results of the present study indicated that post-transcriptional regulatory pathways notably affect the protein expression of DAPK1. Although the DNA methylation of DAPK1 was widely reported in multiple types of cancer, only a few of them examined mRNA expression or the protein expression of DAPK1 (5,11-13). Therefore, extra caution is required when interpreting the prognostic value of DAPK1 DNA methylation, as it may not be associated with DAPK1 protein expression and may actually reflect the activity of other proteins involved in DNA methylation.

It took $\sim 5$ days to effectively knockdown DAPK1 protein expression in MCF7 and MDA-MB-231 cell lines. Whereas in previous studies, exogenous DAPK1 protein had a relatively short half-life and its expression dropped to 10-20\% once DAPK translation was stopped using cycloheximide within $48 \mathrm{~h}$ (14). Furthermore, the knockdown of DAPK1 in colon cancer cell lines using small interfering RNA significantly decreased DAPK1 expression levels $48 \mathrm{~h}$ post transfection (15). These data indicated that the stability of exogenous and endogenous DAPK1 proteins in different tissues is different, which may be caused by the diverse range of binding partners, and cellular environment. At present, DAPK1 is known to be degraded via proteasomal and lysosomal signaling pathways $(14,16)$. The ubiquitin E3s for DAPK1 include mind bomb 1 (16), C-terminus of Hsc70-interacting protein (17) and kelch like family member 20-cullin3-ring-box 1 complex (18). TSC2 and a splice variant of DAPK mediate the lysosomal degradation of DAPK1 $(3,14)$. Furthermore, a lysosomal protease, cathepsin B, is able to cleave DAPK1 in response to tumor necrosis factor receptor 1 overexpression (19). The tissue specific DAPK1 stability control may be due to the differential expression of these proteins. The inducible knockdown of DAPK1 resulted in the reduced viability of MDA-MB-231, but not MCF7 cells, supporting the model that DAPK1 positively regulates the viability in p53 mutant breast cancer cells (6). In addition to p53, a number of components in the ER signaling pathway, including extracellular signal-regulated kinase were reported to interact and regulate DAPK1 activity $(20,21)$.
It is unclear whether the status of these upstream signaling molecules of DAPK1 is different in MCF7 and MDA-MB-231 cells, and contributes to the differential viability responses. Of note, DAPK1 did not function as a prognostic factor for patient survival in the present dataset regardless of the ER status. One potential explanation may be that the patient cohort of the present study was limited in size. There were only patients with TNBC in our dataset and the p53 mutation status was not clear. Furthermore, the scoring system used in the present study is different from that used in a previous publication (6) as patients were classified into negative and positive, which were considered to be least likely to create any confusion. Further studies are required in order to elucidate the clinical function of DAPK1 in breast cancer.

However, the inducible knockdown of DAPK1 did not affect migration in either of the breast cancer cell lines. However, the ability of DAPK1 to suppress the migration of breast cancer cell lines, including MDA-MB-231 has been previously demonstrated (8). This discrepancy may be due to the difference technique used to regulate DAPK1 expression. Previous studies have typically reported a clear migration or invasion suppressive effect of overexpressed DAPK1 $(15,22)$. Although DAPK1 is capable of suppressing cell motility, this function may not be triggered during the physiological stage and requires extra signal stimulation (22). Furthermore, previously, Ivanovska et al (15) reported that DAPK1 did not affect proliferation in colon cancer, but was strongly associated with the migration and invasion of colon cancer cells, suggesting that the physiological function of DAPK1 may be tissue specific.

In conclusion, the present study investigated the ability of DAPK1 to regulate cell viability, colony formation and migration in breast cancer cell lines. Endogenous DAPK1 was only able to regulate the viability and colony formation of MDA-MB-231 cells. Further studies are required to investigate the physiological functions of DAPK1 and the key factors determining the tissue specific functions of DAPK1.

\section{Acknowledgements}

Not applicable.

\section{Funding}

The present study was supported by the Scientific Research Innovation Team Construction Program of Fujian Normal University (grant no. IRTL1702), the Natural Science Foundation of Fujian Province (grant nos. 2016Y0029, 2016J01146 and 2016J01538) and the Key Clinical Specialty Discipline Construction Program of Fujian, P.R. China [grant no. (2013) 544].

\section{Availability of data and materials}

All data and materials generated or analyzed in the present study are included in this manuscript.

\section{Authors' contributions}

YH, YZ and YL were responsible for study conception and design. YH, ML, XC, CH, XZ, LC, KW and YC performed data 
collection and assembly. YH, ML, XC, YZ and YL performed data analysis and interpretation. All authors approved the final version of this manuscript.

\section{Ethics approval and consent to participate}

The present study was approved by the Ethics Committee of the First Affiliated Hosptal of Fujian Medical University, and informed consent to participate in the study was obtained from all patients involved.

\section{Consent for publication}

No identifying patient information is included in the published manuscript.

\section{Competing interests}

All the authors declare that they have no competing interests.

\section{References}

1. Shiloh R, Bialik S and Kimchi A: The DAPK family: A structure-function analysis. Apoptosis 19: 286-297, 2014.

2. Lin Y, Hupp TR and Stevens C: Death-associated protein kinase (DAPK) and signal transduction: Additional roles beyond cell death. FEBS J 277: 48-57, 2010.

3. Lin Y, Stevens C, Harrison B, Pathuri S, Amin E and Hupp TR: The alternative splice variant of DAPK-1, s-DAPK-1, induces proteasome-independent DAPK-1 destabilization. Mol Cell Biochem 328: 101-107, 2009.

4. Lin Y, Stevens C, Hrstka R, Harrison B, Fourtouna A, Pathuri S, Vojtesek B and Hupp T: An alternative transcript from the death-associated protein kinase 1 locus encoding a small protein selectively mediates membrane blebbing. FEBS J 275: 2574-2584, 2008.

5. Huang Y, Chen L, Guo L, Hupp TR and Lin Y: Evaluating DAPK as a therapeutic target. Apoptosis 19: 371-386, 2014.

6. Zhao J, Zhao D, Poage GM, Mazumdar A, Zhang Y, Hill JL, Hartman ZC, Savage MI, Mills GB and Brown PH: Death-associated protein kinase 1 promotes growth of p53-mutant cancers. J Clin Invest 125: 2707-2720, 2015.

7. Stevens C, Lin Y, Harrison B, Burch L, Ridgway RA, Sansom O and Hupp T: Peptide combinatorial libraries identify TSC2 as a death-associated protein kinase (DAPK) death domain-binding protein and reveal a stimulatory role for DAPK in mTORC1 signaling. J Biol Chem 284: 334-344, 2009.

8. Kuo JC, Wang WJ, Yao CC, Wu PR and Chen RH: The tumor suppressor DAPK inhibits cell motility by blocking the integrin-mediated polarity pathway. J Cell Biol 172: 619-631, 2006.

9. Xie JW, Chen PC, Zheng CH, Li P, Wang JB, Lin JX, Lu J, Chen QY, Cao LL, Lin M, et al: Evaluation of the prognostic value and functional roles of CD44v6 in gastric cancer. J Cancer Res Clin Oncol 141: 1809-1817, 2015.
10. Lin Y, Richards FM, Krippendorff BF, Bramhall JL, Harrington JA, Bapiro TE, Robertson A, Zheleva D and Jodrell DI: Paclitaxel and CYC3, an aurora kinase A inhibitor, synergise in pancreatic cancer cells but not bone marrow precursor cells. Br J Cancer 107: 1692-1701, 2012.

11. Lehmann U, Celikkaya G, Hasemeier B, Langer F and Kreipe H: Promoter hypermethylation of the death-associated protein kinase gene in breast cancer is associated with the invasive lobular subtype. Cancer Res 62: 6634-6638, 2002.

12. Christoph F, Kempkensteffen C, Weikert S, Köllermann J, Krause H, Miller K, Schostak M and Schrader M: Methylation of tumour suppressor genes APAF-1 and DAPK-1 and in vitro effects of demethylating agents in bladder and kidney cancer. $\mathrm{Br}$ J Cancer 95: 1701-1707, 2006.

13. Satoh A, Toyota M, Itoh F, Kikuchi T, Obata T, Sasaki Y, Suzuki H, Yawata A, Kusano M, Fujita M, et al: DNA methylation and histone deacetylation associated with silencing DAP kinase gene expression in colorectal and gastric cancers. Br J Cancer 86: 1817-1823, 2002.

14. Lin Y, Henderson P, Pettersson S, Satsangi J, Hupp T and Stevens C: Tuberous sclerosis-2 (TSC2) regulates the stability of death-associated protein kinase-1 (DAPK) through a lysosome-dependent degradation pathway. FEBS J 278: 354-370, 2011.

15. Ivanovska J, Zlobec I, Forster S, Karamitopoulou E, Dawson H, Koelzer VH, Agaimy A, Garreis F, Söder S, Laqua W, et al: DAPK loss in colon cancer tumor buds: implications for migration capacity of disseminating tumor cells. Oncotarget 6: 36774-36788, 2015.

16. Jin Y, Blue EK and Gallagher PJ: Control of death-associated protein kinase (DAPK) activity by phosphorylation and proteasomal degradation. J Biol Chem 281: 39033-39040, 2006.

17. Zhang L, Nephew KP and Gallagher PJ: Regulation of death-associated protein kinase. Stabilization by HSP90 heterocomplexes. J Biol Chem 282: 11795-11804, 2007.

18. Lee YR, Yuan WC, Ho HC, Chen CH, Shih HM and Chen RH: The Cullin 3 substrate adaptor KLHL20 mediates DAPK ubiquitination to control interferon responses. EMBO J 29: 1748-1761, 2010.

19. Lin Y, Stevens C and Hupp T: Identification of a dominant negative functional domain on DAPK-1 that degrades DAPK-1 protein and stimulates TNFR-1-mediated apoptosis. J Biol Chem 282: 16792-16802, 2007.

20. Stevens C, Lin Y, Sanchez M, Amin E, Copson E, White H, Durston V, Eccles DM and Hupp T: A germ line mutation in the death domain of DAPK-1 inactivates ERK-induced apoptosis. J Biol Chem 282: 13791-13803, 2007.

21. Chen CH, Wang WJ, Kuo JC, Tsai HC, Lin JR, Chang ZF and Chen RH: Bidirectional signals transduced by DAPK-ERK interaction promote the apoptotic effect of DAPK. EMBO J 24: 294-304, 2005

22. Kuo JC, Wang WJ, Yao CC, Wu PP and Chen RH: The tumor suppressor DAPK inhibits cell motility by blocking the integrin-mediated polarity pathway. J cell Biol 172: 619-631, 2006.

(i) $($ ) This work is licensed under a Creative Commons Attribution-NonCommercial-NoDerivatives 4.0 International (CC BY-NC-ND 4.0) License. 